\title{
TRATAMIENTO QUIRURGICO DE LA TUBERCULOSIS PULMONAR DEL NIÑo
}

\author{
l'oz los Irs. RAUL MMTIE I. y SERGIO IBANEZ Q. \\ Catcotra del Itof. A. Ariesía A. - Hospital Luis Calvo Mackenna
}

Los estudios epidemiológicos de la thc. en Chile demuestran que nuestra población pasa por una fase de tuberculinización máxinra, o sea que las curvas de morbo-nuortalidad estín prácticamente estacionarias, ocupando la tbe. la segunda causat de todas las muertes ocurridas en Chile. Si it este estado innuno-bioló. gico de nuestru población se agrega que no existe ningún plan integral y racional de lucha y profilaxia de la tbc, que no se diagnostica precozinente la enfermedad, que los focos permanecen largo tiempo en su hagar por falta de sitios apropiados pard aislarlos y tratarIos y que las condiciones higieno-dietéticas y de habitación son muy deficientes, nos explican que la tuberculosis ocupe una de las causas mís importantes en la patología infantil. - 1

La gravedad de esta infección la sido puesta muchas veces en evidencia por nuestros tisiólogos e higienistas, con las curvas de mortalidad y prevalencia de la enfermedad e infec. ción de nuestra población. A este respecto nos parece muy demostrativo y revelador de la realidad cle mestro medio urbano el estudio estadistico hecho entre nosotros por el Dr. Enrique Pereda sobre "algunos aspectos de la epidemiología de la tbc. en la Comuna de Quinta Normal". Como esta comuna representa un barrio obrero particularmente pobre, con condiciones sanitarias muy mediocres, los resul. tados obtenidos no pueden generalizarse a todo el pais, sino que a otras comunas en iguales condiciones. Sin embargo, son representativas por la seriedad y minuciosidad del estudio.

Este autor encuentra que la prevalencia de la infección en el grupo de 0.4 años es de un $43 \%$ de infectados, cifra que se eleva al $88 \%$ en el grupo de 10-14 y prácticamente al 100 por 100 a los 20 años. Estos números indican la precocidad del contagio en los niños.

En cuanto al índice de enfermedad encuentra que alcanza a1 9,5\% en general. Por edades es relativamente alto en el grupo 0.4 con un $9 \%$, baja en el grupo 5-10 años a un 6\% y sube de nuevo en el grupo de cdad que sigue a un $13 \%$.

Nos parece interesante citar tambićn el trabajo hecho por el Dr. Pedro Araya sobre la epjidemiologia de la tbc. del niño durante el periodo 1944-1946. Lin este estudio la tbc. ocupat el 2o lugar con $12,9 \%$ del total de muertos por todlas las causas.

Si nos circunscribimos a la infancia la tbc. ocupa en los niños de 1-4 años el 3.er lugar con 7,8\% del total de muertos; pasa al $2^{\circ}$ lugar con 20,7\% en los niños de 5-9 añas y al primer lugar con el $32,3 \%$ del total de muertos en los niños de 10-14 años.

Del total de muertos por tbc. (13.110 fallecidos) el $17,5 \%$ se produce en los niños antes de cumplir 15 años $(2.300)$, o sea, que esto demuestra que la infancia paga un fuerte tributo a la tuberculosis.

Estamos convencidos que estas cifras de morralidad son muy inferiores a la realidad, ya que se basan en datos proporcionados por la estadística en que las causas de muerte son muchas veces imprecisas y en otras ocasiones no son hechas por médicos sino por testigos no médicos, o por funcionarios médicos de sanidad que no conocían en vida al paciente. Sabemos Ia dificultad para hacer el diagnóstico de tbc. sobre todo en aquellas regiones o zonas rurales en que se cuenta con los medios de diagnóstico apropiado.

Hemos creido de interćs traer estos datos estadísticos por cuanto ellos nos demuestran la gravedad que tiene en la infancia la tbc. Pric. ticamente podemos decir, que en nuestro medio urbano una gran parte sle los niños se ponen en contacto con el bacilo de Koch des. de muy temprana edad y que ya a los 20 años todos están infectados. Esto fuera de demostrar la fase epidemiológica por que atravesamos nos pone de manifiesto que nuestro armamento antituberculoso es primitivo. No hay aislamiento de focos, el diagnóstion se hace en forma tardia y no hay los medios suficientes para su tratamiento adecuado. Todo esto nos explica por qué nuestros hospitales infamtiles se ven asediatos por niños tbc., sus secciones Broncopulmonares se hacen estrechats y clia a día rechazamos hospitalizaciones de formas curables cle the. También nos explica que formas de the. infantil benignas que tratadas convenientemente son todias curables, se transforman por las malas condiciones de sus longares que no 
les pueden ofrecer las condiciones mínimas de vivienda, higiene y alinentación y por las $\mathbf{s u}$ perinfecciones repetidas, en formas graves con grandes destrucciones de sus parénquimas y con generalizaciones que hacen que su pronústico de benigno que era se transtorme en grave y que si curan lo hacen dejando grandes residuos, crmpos de induración, que van a ser el punto de partida cle la tbc. del adulto.

Se sabe que curar una infección primaria en luenas condiciones constituye la mayor garantia para que ese individuo no haga lana tbc. en la culad adulta.

Veamos ahora qué es lo que observamos en nuestro hosyital Calvo Mackenna referente aI problema de la tbc. infantil.

De 23.740 niños ingresados desde su inaugruración en jutio de 1942, en 1.800 la causa de su ingreso fue una tbc. activa.

En este periodo fallecieron 1.801 niños entre los cuales 328 murieron por tbc, o sea que cada 5 niños muertos, uno muere por causa de la the.

En nuestro servicio de Broncopulmonares han pasado desde 1942, 931 niños tbc., seleccionados en el Policlinico de este hospital. De estos 931 niños 597 presentan formas benignas de la tluc. infantil y fueton dados de alta en buenas condiciones, en cambio 344 se les diag. nosticó formas graves. Llamamos formas graves todas aquellas que presentan un carácter evolutivo franco, va sea del foco pulmonar o ganglionar, con cenclencia a la caseificación y a la diseminación. Generalmente son abiertas, con eliminación de bacilo de Koch y que dejaclas a su curso natural fallecen gran parte de ellas.

De estos 344 niños fallecen en el hospital 117 casos y se han dado de alta con sus lesiones inactivas 227 .

Esto nos estí indicando gue el pronóstico inmediato de las formas graves abiertas es nuy desfavorable ya yue en nuestro hospital fallece más del $30 \%$ de los casos.

Es de todos conocido que la tbc. es una enfermelad cronicat que exoluciona por pousces y que por lo tanco para hablar de curación se exige una larga observación. Es así que los atuctes alematnes, suizon y suecos para referirse al pronóstico de una forma grave de tbc. infantil exigen una observación minima de 10 años. Antes no se puede. según cstos autores, decir que un proceso cstát absolutamente sano. Es muy frecuente en nuestro hospital, ver que un niño que ha estado hospitalizado con una forma grave y que hemos dado de alta inactivo, aparentemente sans, vuelva con una nueva reactiyación wás grave, muchas veces incurable, u otras veces tenga graves secuelas consecuencia de su proceso tbc. curado en forma defectuosa.

Nuestro periodo de olservación es todavia reducido para dar cifras estadisticas referentes al pronóstico de estas formas. En todo caso, creemos por lo visto hasta ahora, que nuestras estadísticas coinciden con las de los autores extranjeros que se han ocupado de este problema.

Hat sido especialmente Kurt Klare, Jefe del Sanatorio de niños Scheidegg-Allgäi el que más ha estudiado el problema de pronósticos de Ias tormas abjertas graves, de la tbc. infantil.

Este autor estudiando el porvenir de $436 \mathrm{ni}$ Iios y jóvenes que halsian estado en su Sanatorio entre los años 1926.32 encontró en el año 1936 que ol $75,2 \%$ de estos pacientes habian muerto. Porcentujes parecidos publican tamIsicn Behrendt, Ellinghause, Geissler, Kreutzer y ntros.

Brügger, Jefe de un Sanatorio de Niños de Wangen, Alemania, relata 600 casos con un período de observación (Ie 5 y medio a 16 y medio años, encuentra una mortalidad del $57 \%$.

Simon de t75 cases que estuvieron en su Sanatorio de Aprat, Alemania, entre los años 1915-23 encuentra clespućs de una observación cle 4 a 12 anos una jnortalidad del $85 \%$. En publicaciones posteriores Simon encuentra de5pués de un tiemplo de observación de 10-13 años una mortalidad de $9,5,1 \%$.

Burchhardt de Davos (citado por Klare), entre 118 niños observados de 9 a $9 \mathrm{I} / 2$ años encuentra un $52 \%$ de mortalidad.

Wilkelmann de Sachsen sobre 154 niũos observados entre $1936-16$ en su Sanatorio y con una observación de 3-13 años tiene una nortalidad de 67 a $79 \%$.

Widmer en un trabajo recićn publicado en da revista suiza de thc. obtiene una mortalidad de $48 \%$ en la tuberculosis infantil abierta y un $61 \%$ en las que tenian caverna. clespućs dé 10 años de observación (1).

Del análisis de todos estos datos estadísticos se desprende la gravedad que presentan los procesos tbc. en la intancia, cuando éstos de benignos que son cuando se inician, se translorman en formas abiertas graves. Nucstros esfuerzos deberán clirigirse especialmente a evitar este proceso, a tomar todas las medidas profilácticas que la experiencia aconseja para

(1) Futre nosotros eI 1)r. Roberto Infante hat relat cario resultaclos parcciclos, pero en un plazo más corto. 
impedir que esto suceda. Desgraciadamente nuestra realidad es distinta y tenemos el triste privilegio de recibir constantemente en nuestras salas, especialmente nin̄os veniclos de medios alejados rurales, formas graves, avanzadas de tuberculosis en Ias cuales y: se han producido procesos destructivos irreparables en sus pulmones. Muchas veces se logra con todos los métodos modernos de tratamiento cle colapsolerapiat, antibióticos, detener el proceso e inactivarlo y muchas veces que se rodee de tejido fibroso. Sin embargo, el pronústico alejado de estos casos sigue siendo malo, ya que han curado dejando grandes residuos que son reservorios cle bacilos de $\mathrm{Koch}$ que se reagudizarán en cualquiera ocasión que ese organismo se debilite. En otros cisos es malo el futuro porque esos procesos cicatriciales extensos provocan grandes alteraciones mecánicas del mediastino con las consiguientes molestias cardiovasculares (fibro-tórax) o bien procesos extensos de dilataciones bronquiales con todas las consecuencias conocidas.

Es por todas estas razones que hemos acogido con entusiasmo, en nuestro hospital, los grandes adelantos que ha experimentado la cirugia toríxica en estos últimos años. Ella con el conocimiento exacto de la anatomia de los vasos y bronquios, como su distribución en segmerítos y la anestesia intratraqueal ha hecho posible la extirpación de las partes destruidas de Ios pulmones, sin riesgos mayores.

En la tbc. del adulto, estos métodos yat han sido experimentados a larga mano y podemos decir que están incorposados en su terapéutica habitual. Se conocen bien sus indicaciones, contraindicaciones y complicaciones, lo mismo que sus resultados inmediatos y alejados. No podemos decir lo mismo en lo referente a la tbc. infantil. Su aplicación es reciente, no se conocen bien sus indicaciones y sobre todo no se sabe cuáles son sus resultados alcjados.

En la literatura extranjera no hemos encontrado, que nosotros sepanos, experiencias al respecto, solo hay cierta experiencia con toracoplastía, que en general no son partidarios los autores, por las deformaciones que produce. Referente a resecciones pulmonares en thc. activas no hemos encontrado literatura, sólo se han practicado en casos de bronquiectasias y malformaciones quísticas congénitas de los pulmones. En todos estos casos los niños, aun de corta cdad, han demostiado una gran tolerancia a estas intervenciones, resistiéndolas en mejores condiciones que el adulto. Es por cste motiro que nos la parecido interesante dar a conocer nuestra experiencia en el tratamients quirúrgico de la tbc. pulmonar del niño.

Todo nuestro material ha sido seleccionado de niños hospitalizados en el Hospital Calvo Mackenna y en los Sanatorios Martínez de Feruari y San Luis y Susana Palma.

Las intervenciones han sido hechas en el servicio de Cirugía de este Hospital, primero por el Dr. A. Alonso y la mayoría por los Dres. H. Salvestrine y Jaeger y la anestesia dada por cl Dr. Jaime Huidobro.

La técnica quirúrgica y anestésica ha sido la usual en estas interyenciones y no entraremos en detalles ya que no es de nuestro resorte.

Despucis del postoperatorio todos estos niños lan permanecido largo tiempo en observación en los Sanatorios antes mencionados, para ser tados de alta a sus casas cuando ya su curacion no ha merecido duda. Posteriormente han sido controlados en nuestros Policlínicos.

Nuestro material se compone de 47 casos de los cuales 31 corresponden a intervenciones hechas en tbc. activas y 16 a procesos cicatriciatles inactivos consecuencia de un proceso tbc. que ha curado en malas condiciones slejando grandes bronquiectasias. La edad de los niños osciló desde los 2 años 4 meses a los 1.1 años.

Indicaciones: para buscar las indicaciones nos hemos basado en la experiencia de los cirujanos y tisiólogos de adultos, donde ya se conocen ron más o menos precisión y por otra parte en los conocimientos que hemos adquj. rido del pronóstico de las clistintas formas de la tbc. infantil.

Desde luego queremos dejar establecido que liemos indicado la cirugía del tórax especialmente la toracoplastia y resecriones, solamente en las formas graves, especialmente cuando éstas van acompañadas de extensás destrucciones del parénquima en forma de focos caseosos, fibrocaseosos o cavernas irreparables y siempre después de haber fracasiklo en el tratamiento con el uso de la colapsoterapia y antibiótico.

Nos teferiremos separadamente it las distintas formas de intervención que se han practicaclo en este hospital.

\section{Toracoplastia}

La indicación cle esta intervención la constituyen las lesiones cavitarias unilaterales, suprahiliares, de tipo predominantemente fibroproductivo o exudativa estacionaria o regresivi, en formas que responden bien al tratamiento médico. 
Estará contraindicada cuando las cavidades sean de tipo que no se dejen comprimir, ya sea por paredes fibrosas o bien por ser insufladas por defectos del bronquio de dremaje, obstruccion bronquial con atelectasia. Las bronquiectasias también constituyen una mala indicación.

No se debe hacer toracoplastia si hay una lesión no estabilizada por el reposo en cama a una lesión activa contralateral o exacerbación reciente; lo mismo sucede cuando hay una lesion activa extrapulmonar. Contraindicación relativa la constituyen las cavernas suprahiliares pegadas a los canales vertebrales que no se dejen comprimir.

En Pediatria, en general esta intervención ha tenido poca aceptación por las deformaciones que provocan, en organismos que están en pleno crecimiento. Fs por este motivo que estaria indicada sólo cuando ćste ya ha terminado su desarrollo. Nosotros la hemos indicado en 8 casos, la mayorla en niñitas de 13 a 15 años.

En niños menores en quc está indicada esta intervención los cirujanos han preferido hacer neumolisis extrapleural con plombaje de esferas de lucita (material plástico). En esta intervención basta con resecar un segmento de costilla para desprender el vértice y colocar las esferas de lucita. También prefieren estas intervenciones en lesiones apicales estabilizadas, en que no se puede hacer otro trataniento por ser lesiones bilaterales.

\section{Resecciones pulmonares}

Para los tisiólogos de adultos las indicaciones se podrian dividir: primero en absolutas o indiscutibles, segundo discutibles, tercero heroicas.

Entre las absolutas tendriamos: $1^{0}$ tbc. bronquial estenosante crónica. Este proceso al producir atelectasias totales o parciales con los signos consiguientes de grandes retracciones del metiastino y fibrosis permanentes con bronquiectasias crónicas conducen a situaciones catastróficas de la función respiratoria y alteraciones vasculares.

En los niños esta obstrucción bronquial pue. de producirse ya sea porque el foco primario está situado én la pared bronquial o en la vecindad produciendo una alteración bronquial desde su iniciación o bien por el foco ganglionar que irrumpe en el bronquio com. prometiéndolo y obstruyóndolo.

Estará indicada la resécción sólo en estos ca. sos en que la lesión bronquial es irreparable.
Se descartarán las atelectasias pasajeras por lesiones bronquiales inflamatorias, que en el curso de algunos meses desaparecen y se restituye la permeabilidad bronquial.

29 Tbc primarias o postprimarias graves o de reinfección que esté bien indicada la colapsoterapia y ella fracasa por diferentes causas como adherencias incontrolables o colapsos atelectásicos por lesiones bronquiales.

3 . Cavernas que no se colapsen por el neumotorax o frenoparálisis ya sea porque sus paredes sean rígidas o porque su bronquio de drenaje no funciona. EI mismo problema lo presentan ciertas cavernas mal colocadas, como las que se sitúan en el vértice del lob. inf. a pesar de que para algunos esta seria una indicación discutible.

4 a En caso de neumonía caseosa tengan o no tengan caverna en que los tratamientos colapsoterápicos no dan resultados.

50 Procesos caseosos cavitarios superficiales cuya caverna se perfora a la pleura, ya sea en el curso del neumotórax o por otras circunstancias y que dan lugar a empiema crónico. Estos casos constituyen una indicación de urgencia como lo demuestra un caso que presentaremos.

$6^{\circ} \mathrm{Y}$ por último constituyen otra indicación de resección los procesos residuales de una thc. primaria, ya sea en forma de tuberculomas, o sea focos primarios tumorales caseosos o conglomerados de tubérculos rodeados de una gruesa capa fibrosa o bien en forma de campos de induración con focos múltiples conglonerados calcificados que por su exten. sion y constitución anátomo-patologica constituyen un permanente peligro para la vida del níno, ya sea por frecuentes reactivaciones o zona de menor resistencia o bien porque dan lugar con frecuencia a procesos lronquiectásicos donde la indicación de resección es perentorla.

Como indicaciones heroicas los tisjólogos de aclultos indican las tbc. malignas en que fra. casan todos los tratamientos y las hemoptisis graves.

Ifuy importante nos parece para hacer una buena indicación, el estudio minucioso del lado opuesto. Si para la toracoplastia se exige que las lesiones sean unilaterales, para las resecciones esta condición debc ser más estricta. En caso de lesiones nodulares de diseminación eti el lado opuesto, como son varios de nuestros casos, debemos exigir que éstos estén inactivos, que sean lesiones duras fibrosas, que no den signos clínicos de actividacl. 
Hoy día con el uso de los antibióticos se han ampliado considerablemente las indicaciones de estas intervenciones, en el sentido de que procesos que erin antes inoperables o cuando se efectuaban se complicaban de reactivaciones ipsolateral o contralateral, por presentar nódulos en el lado opuesto, hoy gracias a estas drogas se pueden controlar perfectamente con su uso un tiempo antes y después de la intervención.

Todos nuestros enlermos recibieron estreptomicina y PAS antes y despuses de la operación $\mathrm{y}$ en nuestros últimos casos tambion hemos usado la hidrazida del dicido isonicotinico.

En enfermos con lesiones contralaterales sospechosas de actividad y cratándose de casos en que está indicada la toracoplastia se prefierc esta intervención a bien si son niños de menos de 12 años neumolisis extralacial con plombaje de lucitas.

Con el uso de los antibióticos intes de la operación no sólo controlamos el lado opuesto cuando hay lesiones, sino que actuamos sobre el proceso que se ya a extirpar, apagándolo tratándolo de circunscribirlo, disminuyendo las secreciones bronquiales, hechos todos tle vital importancia para que el acto operatorio tenga menos riesgo y sea más sencillo. Por otra parte debemos tratar de que se Ilegue a la intervención antes que los gćrmenes se hagan resistentes a ellos y en todo caso reservar algún antibiótico corno el ácido isomicotinico para dias antes y el postoperatorio. Con estas precaucio. nes habremos obteniclo el máximo de garantias de éxito.

\section{Resultados y complicaciones}

Como lo expresamos anteriormente para poder hablar de resuitados en tbc. se exige un largo periodo de observación. Nuestra experiencia es todavía muv reducida y en especial nuestras observaciones no tienen el tiempo suficiente para poder sacar conclusiones lefinitivas. Sin embargo, porlemos hablar de resultados inmediatos y it un plazo de 2 meses a 3 años y medio, que son los primeros casos intervenidos. En todo caso el resultado es interesante y significativo por tratarse en todos nuestros ninios operatos de t]oc. muy graves cuyo pronóstico era en lat mavoria de ellon fatal a corto plazo.

\section{Casuisticat}

Nuestro material se compone de 47 casos, 31 son the. activa $y$ lo inactiva. Hombres 17 y mujeres 30. La edad fluctuó entre 2 y 11 años. De los 47 fallecieron 6 , siendo 5 del grupo de los procesos activos y uno inactivo.

Nuestra casuistica analizará sólo a los procesos activos. Los separaremos según el tipo de operación.

$1^{0}$ Toracoplastia y neumolisis extraftemal.

lin este grupo hay 10 casos, 8 de toracoplastía y 2 de neumolisis extrapleural con plombaje de lucitas.

Cinco de los casos corresponden a tbc. pul. monar moderadamente avanzada, activa, fibrocaseosa con caverna. Se describen en forma escuemática en la Limina N? I detallando los nombres, edad, diagnóstico pre-operatorio, tipo de operación, conplicaciones, tiempo de observación y estado ictual.

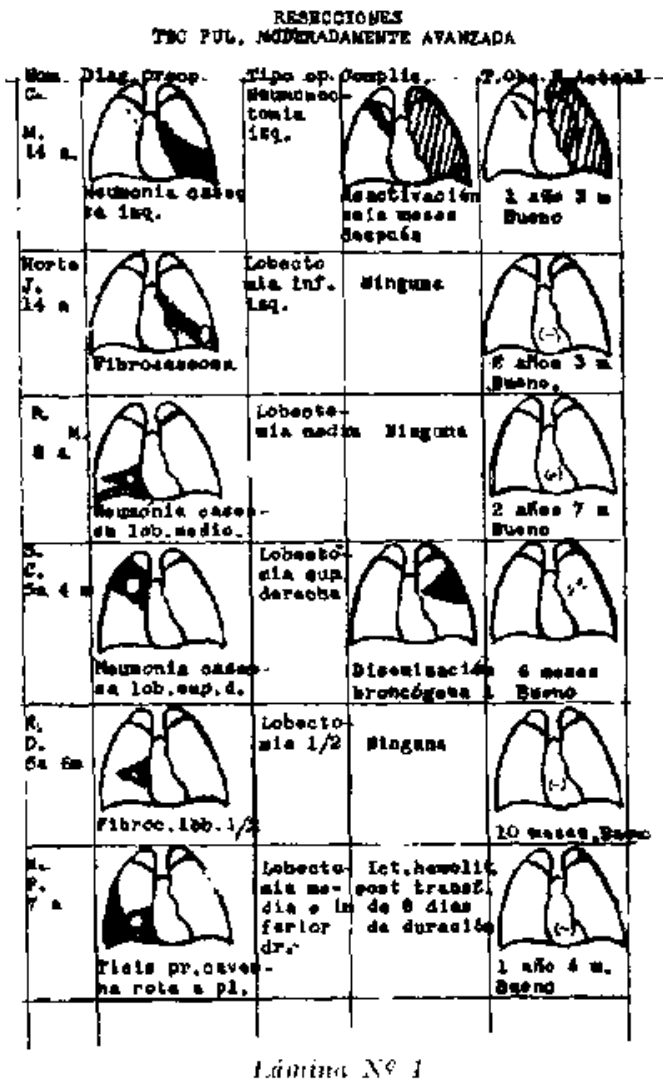

En la Lámina No "2 se describen los otros 5 casos que corresponden a tbc. pulmonar avanzada fibrocaseosa con caverna $y$ diseminación contralatera! que se logró detener y controlar antes de la operación.

El resultado inmediato ha sido muy satisfactorio; los 10 niños están vivos y en buenas condiciones, no habicndo presentado ninguno 
reactivaciones ni complicaciones. El periodo de observación en 3 casos es de más de 3 años, en 3 más de un año y en 1 menos de un ax̃o.

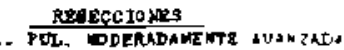

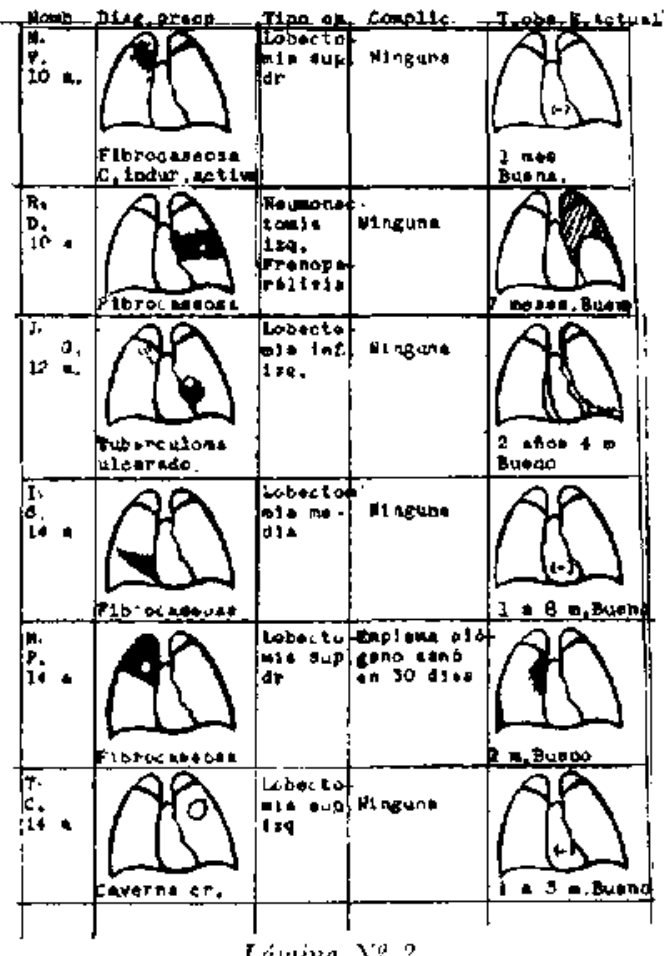

Como todos erin niños de más de 12 años no han presentado deformaciones toráxicas dignas de mención. Su mediastino y columna han permanecido sin alteraciones.

Fn Jos 2 niños que se le hizo neumolisis extrapleural con plombaje de Iucita el resul. tado también ha siclo sitisfactorio hasta el momento. Han tolerado perfectamentc las esferas y sus lesiones estin colapsadas y sin dar signos cle actividad. Se describen 2 observaciones.

Marfa James, Olss. 45; 1697 . liclad: 13 años. Ittgyesat al Iospital por primera ves el 26 de octubte del t.

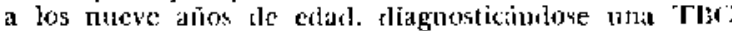
pulimonat postprimaria álcerocaseosa del lóbeto supe-

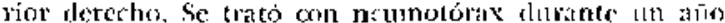
suspendichdose por derrame pleural. Se enria al Sit.

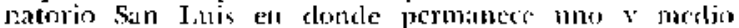

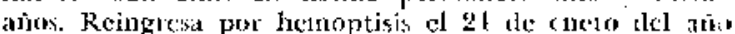
49, diamosticandos: Inx $T B C$ pulmomax de reinfec-

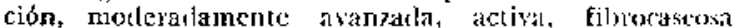
con dos cavernas en el lóbulo superior alerecho. Korl, demostraclo (radiografia $N^{\text {To }} 1$ ).

Se practica frenejutalisis lerceloa sin exico. tho cura com tif grs. de listreptomicina Irgra tocjorar a estado general $y$ ceducir el proceso infiltrativo. sin influir sobre: las carernats, Beoncoscopia normal.
Se inclici conacoplastía por pratarce de una lesiún des. tructira en gila habian fracasarlo lodos los tratamien.

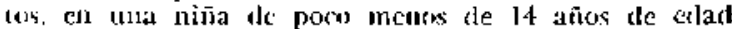
com su lesión localizatla en el lóbulo superior.

El primer tiempo 1a, $2^{* a}$ y $g^{a}$ costilla el ll de jix

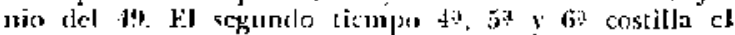
25: ale jutrio del t9.

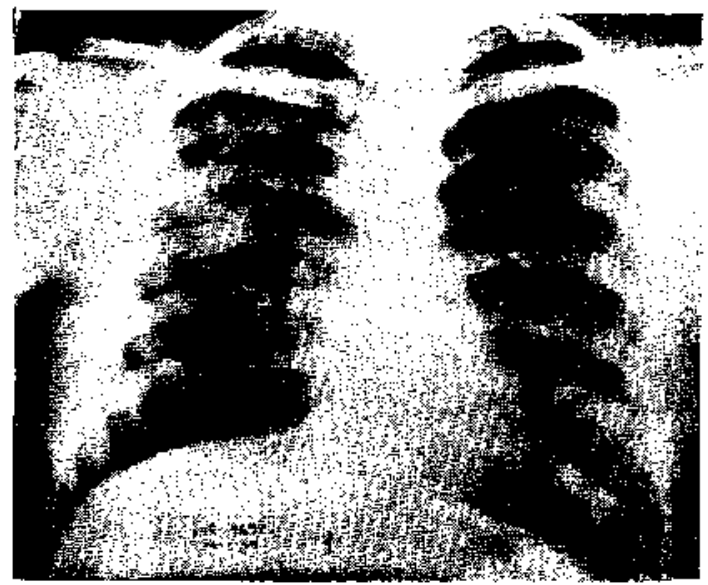

Rendiogrefias No 1

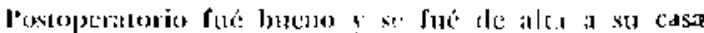

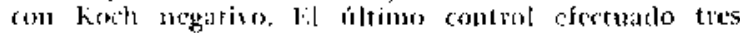

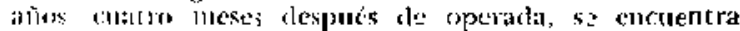

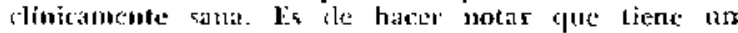
hijo de lacs meses de ctacl y gube ('l cmbarazo y la

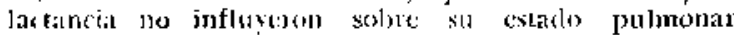

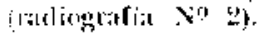
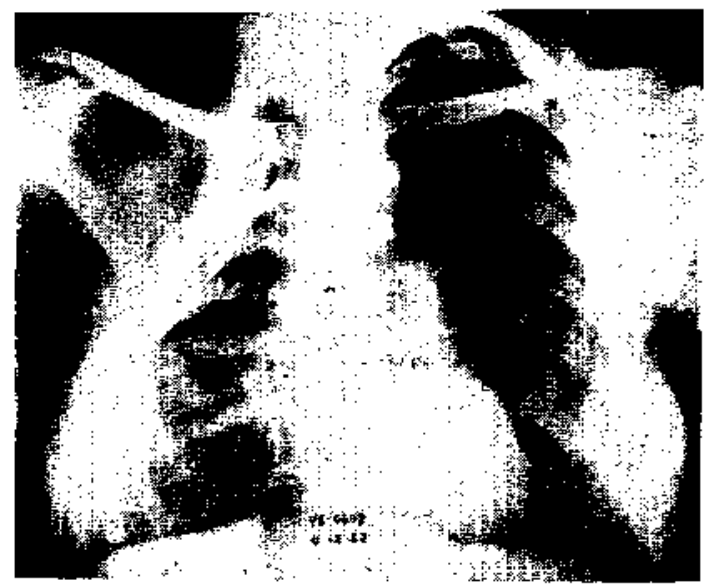

Radingrafia $\mathrm{N} ? 2$

Maria Riveros. Ols, 4ti440\%. Ledad: 7 añes 7 meses.

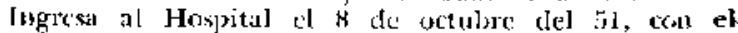
diugnóstico te TBC pulmonat alc rcinfección mote. laclamente avanzata, fibrocascusa, con caverma del lóbuto stuperior dereclio. Koch denesstrado (itcliografia No 3 : planigrafia $\lambda^{\circ} 4$ ). 
Se trata con $63 \mathrm{grs}$, de listreptomicisn obsericuldo discreta reducción. Neunoligrax fracasó por falla de cámala.

En este caso estando indicada la turacoplastí., cul vista de la corla etlad. se prefiere praticar solo luta
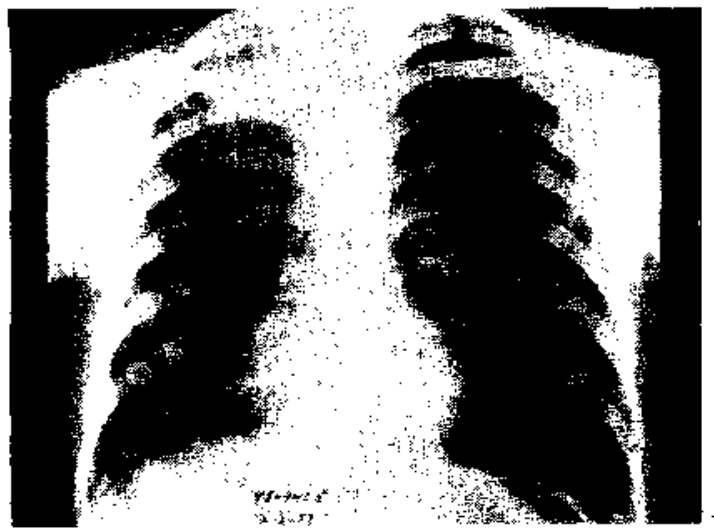

Radiografia No

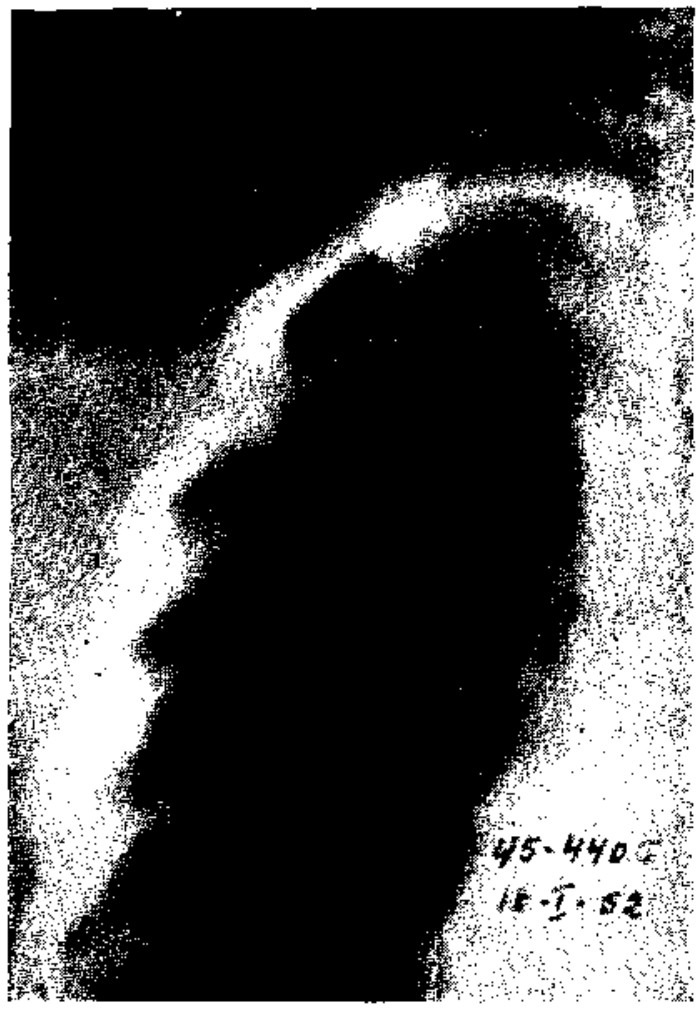

Planigrefiat $\mathrm{X}^{\circ}$

plastia posterolateral cle la segunda, tercera y cuarta costilla con liberación le ta cara inferior cle 1a primera costilia y se colocan cinco pelotas de Lucilas.

Postoperatorio satisfactorio, evulución posterior buc. ma. Fstí aclualmente cu el Sanatorio Snn Luis. Koch negativo. Sudimentación normal. Curva de peso ascendence. I a bdiolugia demuestra un colapso del tencio siperior dol pulnien derecho producido y mantanido por las Ilucitas. Tienipo de observación: ocho meses (1) acliografia No 5).

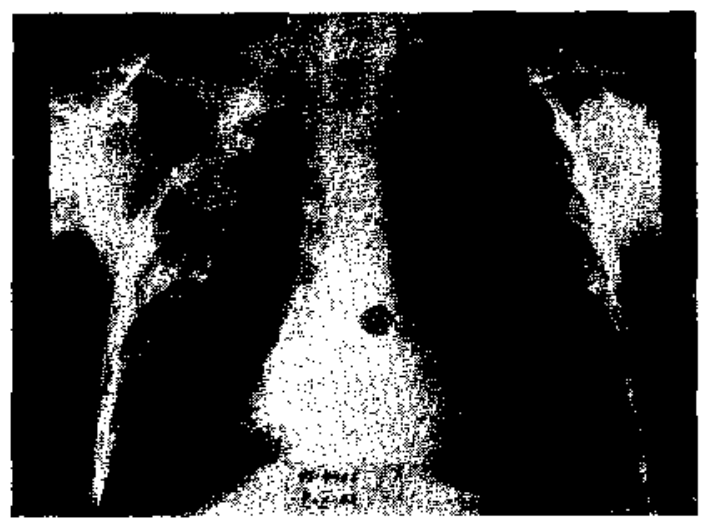

Rarlioguafin X? 5

\section{Resecciones.}

Comprende 21 casos, de éllos 5 fallecieron, los 16 restantes los separamos en: 12 con tbc. moderadamente avanzada (Iáminas N.os 3 y 4) y 4 tuberculosis avanzada (Lúmina No 5).

Se practicaron 7 neumonectomias, 4 a derecha y 3 a izquierda. De estos 7 fallecieron 2 .

Se practicaron 13 lobectomias, 8 a derecha y 5 a izquierda, fallecieron 2.

RESECCION,

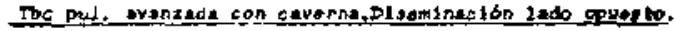

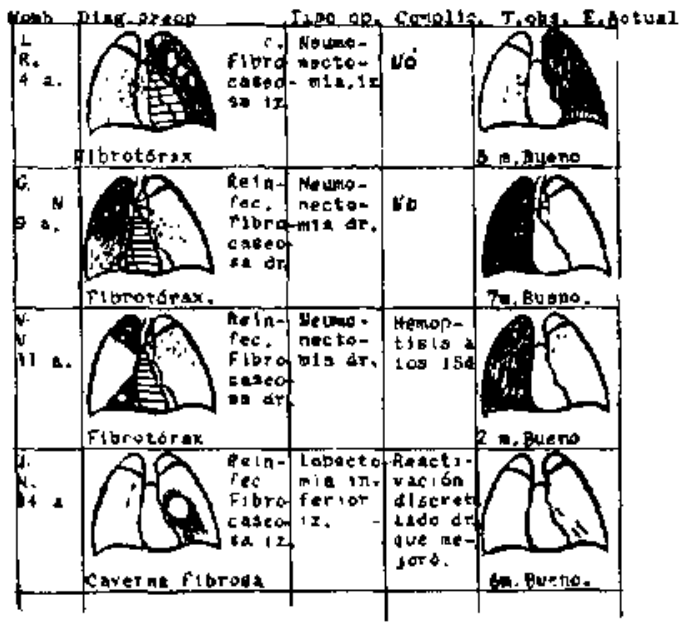

Lamina $N^{0}$ 
TRATAMIENTO QTIRURGICO DE LA TBC.-Drs. Rail Matte Y' 5erglo Inanez

TORACOPLASTIAS

TQC, PUL. MODERADAMEVTK AVAKZRAA.

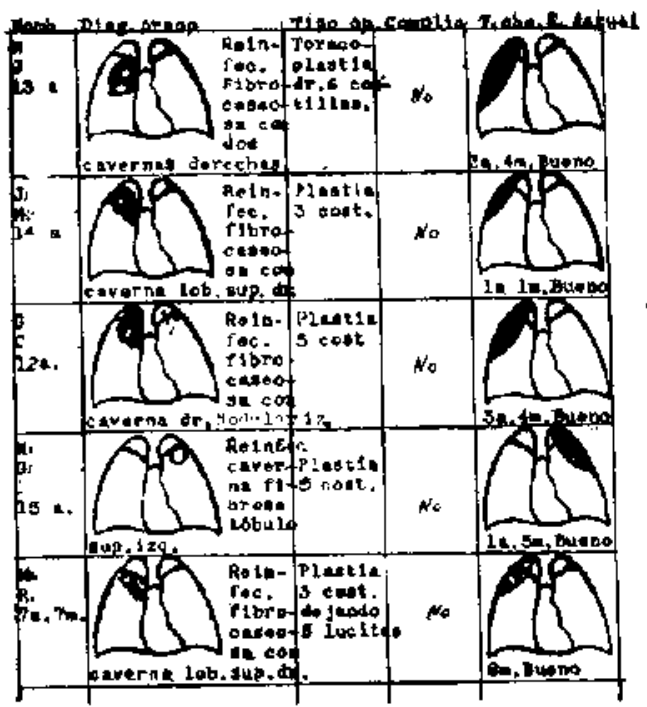

Lamina $N^{P} 4$

\section{TORNGOTLSTTA:}

THC PUL, AVIrUADA.

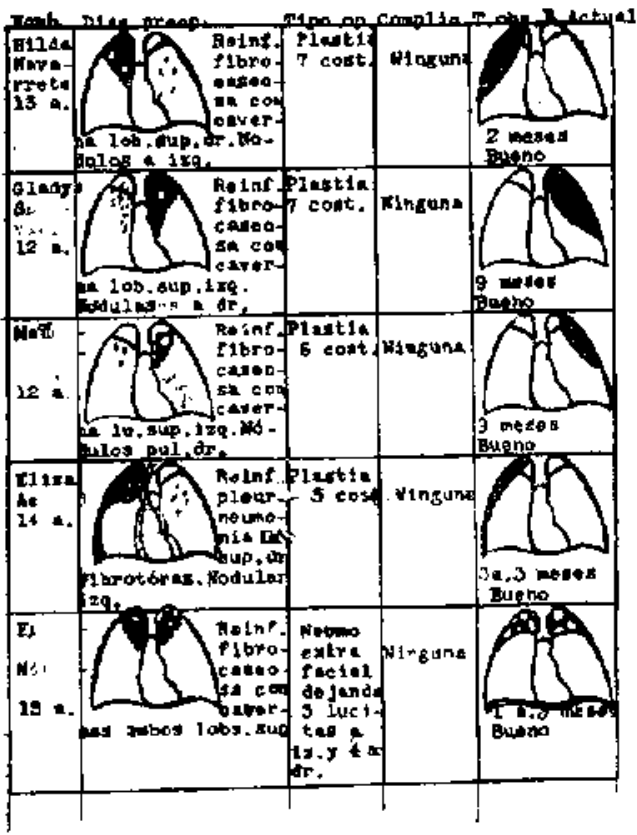

Lamina No 5

En los 5 fallecidos 4 fueron en el postoperatorio inmediato y uno a los 6 meses por una reactivación ipsolateral con fístula broncocutátnea y diseminación lado opuesto.

Se describen con detalle 4 observaciones.
Gaillomos Navirno. Obs, 51/3262, Falad: 9 años. Ingresa al llospital el 3 de julio del jl con el diagnóstico le - I'BC: pulmonal de reinfección. atanzada, hilateral. activat. Hibrocasersa con tavemas y bronquicctásias lúbule tuperior delecleo. Jiseminación nnGular del resto ale aunber campos. Fitrotórax con re tracción de: Ja lovic|tea ? medliasting a derecha. Koch

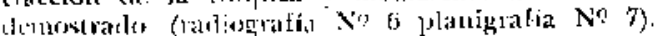

Se trata con listreptomicina 88 gis.. netumoperito.

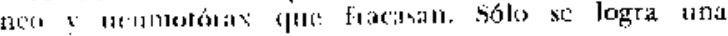

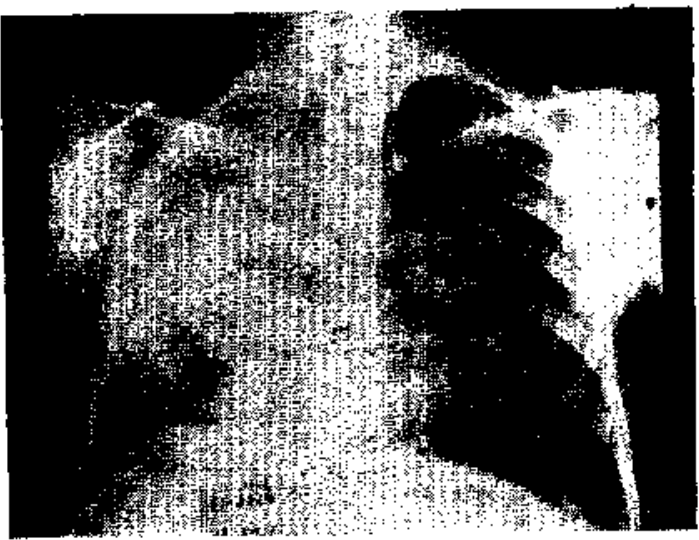

Radiografia No 6

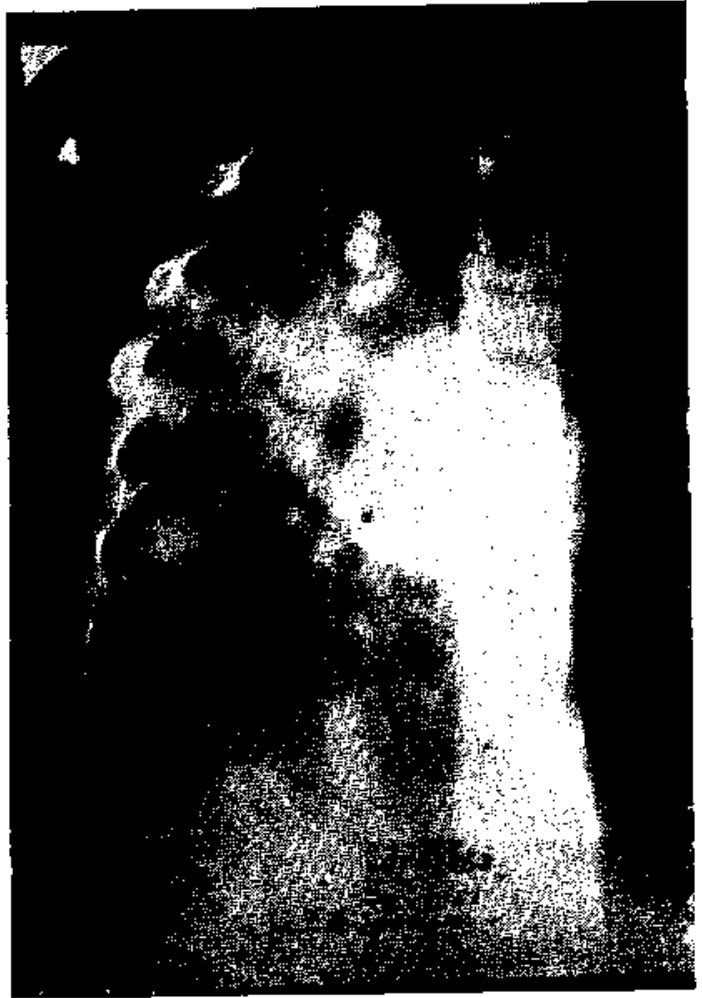

Plonigrafia No 7 


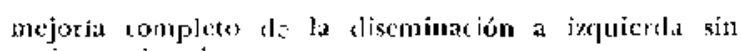
variar a derccha.

Se practica niumoncromia dercha el 20 de maizo dei 52. Sn postoperatorio fut bueno do presents complicaciones, El examen histoparológios demosiró uns tuberculosis fibrocascosit mal, con brinquiestasia (u cl lóbulo superior d:rechos.

Alta al Sandurio San Luis el 5 de jumio clel 52 ca donde permanece al la fectit con su pulmón irpuierdo dinica y adiologicamente sano. Mediastiro y corarón discreanente: lessiarlo a clerceta fradiogralid Nis 8 ).
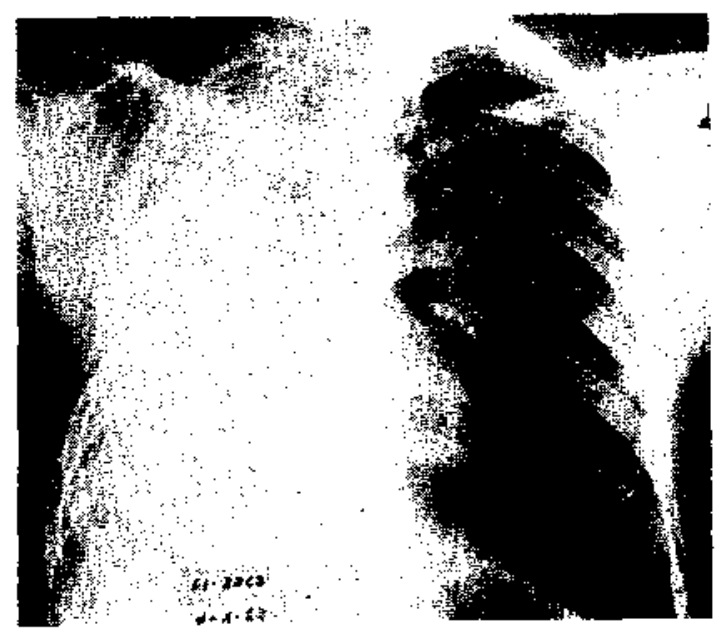

\section{Ratiogufin $x^{\prime} s$}

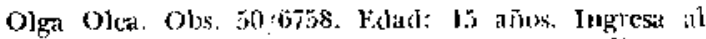
Hospizal el 27 de dicicturtive (le 1950 con el cliagnio: tico de TBC: puluronar postprimaria tinoderadainetr-

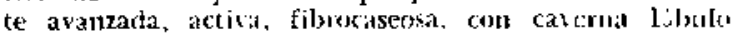
inferior izquicrdo. Foch dementmalo cuablografiá N!! 9 ).

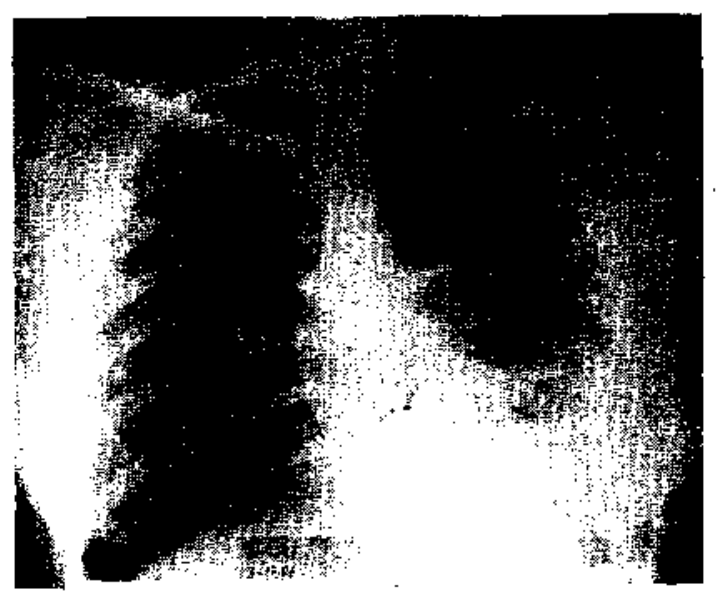

Radiografia No
St lrsión rlala sic tres años autes feclua en Ia cual sc inticia con un infiltado perihiliar iafuicrdo que se cxcita. Se trajj con bistreptomicina ell tos curas; una de 60 grs. Y la otra de 9.9 grs. Nelımotólax fracasa por falta de cínty. Frenoparílisis infficaz. Comn continuals sil lesin en forma cvolutiva s: practica el I4 de marzo del 51 una lobectomia inferiot ispulerda. En la operación se palparon algunes nólulos en cl lisbesto superior.

Su postoperatorio fué malo; reaclivación en el lolsulo superior izquicrdo y diseminación del lado opues16 cn forma precos tiadiognfía N: 10). A los tres meses apareció una fiłcta broncocistínea enpeorando rapidamente. Como ura medida heroica sc trato de reserar al lobulo superior izcuiterdo, pero se encuentra tuna cascosis total icl pulmćn, plenra $y$ tejidos recijids. razón por la enal se hace una plastia.

labece sietc meses despuŕs de la pjiblera inter. varción.

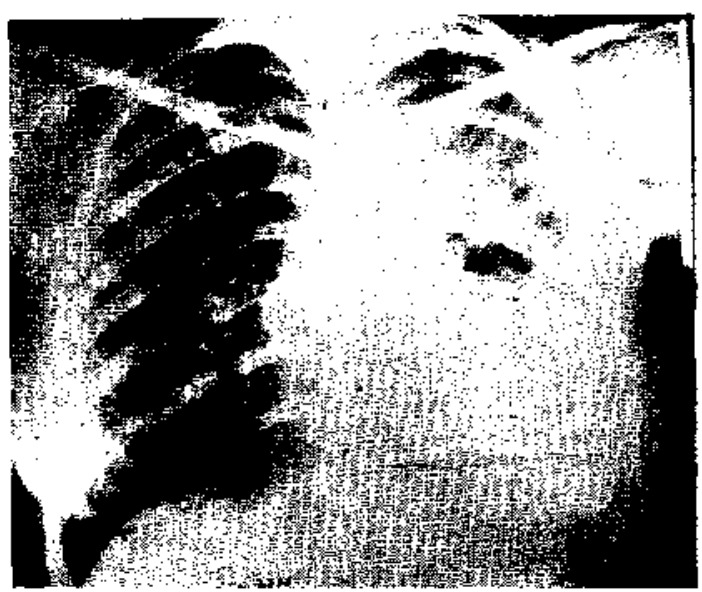

Radiografia $N^{\prime \prime}$,

Manucl P'jnda. Obs, $51 / 2711$. Edad: 7 aios. Ingre. s:1 al Hospital el 8 ele junio ale 1951 con cl diagnóstico de $\mathbf{T B C}$ pulmonar primaria grave, morlerarlamente avanzada, activa. Infiltracién dic todo cl bobulo medio con una gran cavcrna. Diseminación modular en cl lóbulo inferios (leredos, hoch demoscrato (plasigrafía No $1 \mathrm{I}$ ).

Se inicja tratamicuto con Estrepenmicina y diez slias después se practica una broncoscopia con el objelo de conorer cl estado bronguial antes de iniciar neumotórax. Al clia siguicote se agrasa bruscamente por rup. utra de la caverna a la pleura (nadiongrafiat 2 ' 12). Antc estc accidente sc intervienc le urgencia ol 2 th de junio del jil practicándose una lobectomía ak medio e inferior ilerctho. Sc conconeró III hemotórax te 200 continuetros cúbicos. Su pustoperatorio fué bucno.

F.l examen histopatológire revtlo una 13C tilccrocascosa del bobulo medio on una caverma del tamaino te una nuez. Nódulos filurocaseosos en cl lólbulo inferior atcrecho. l'leuritis tuberctzosit fibtinohemonígic3. A]ta a su casa después de scis mese's en Samatorio. Contjol actual: ull ano cuatro meses despucs do la operación cati cano (radiografía No 13 ). 


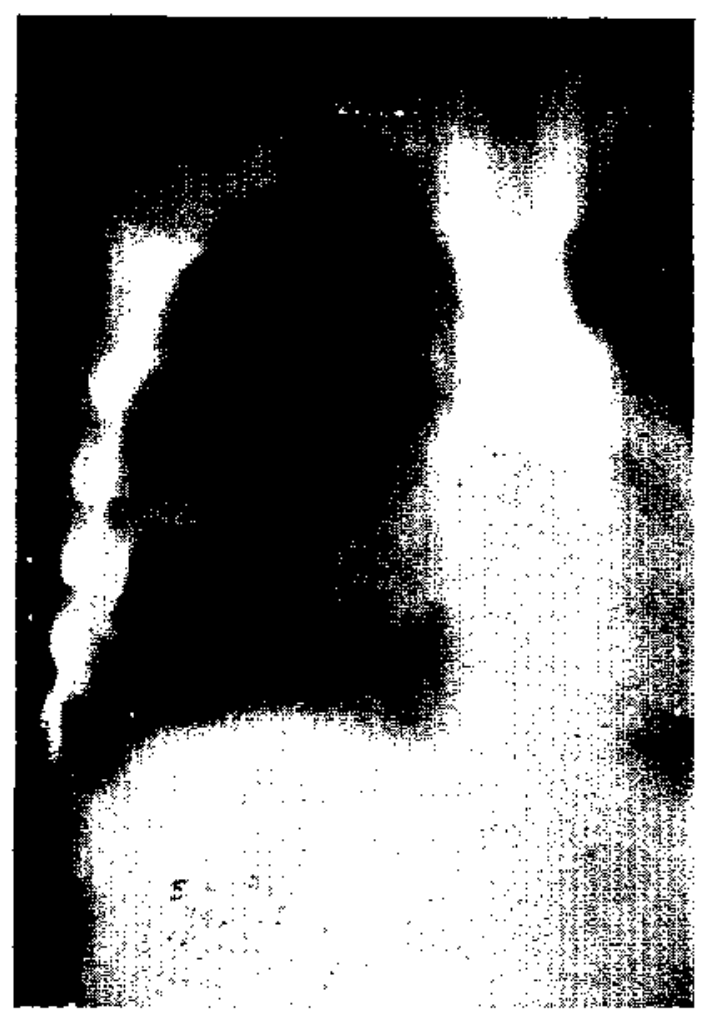

Planigrafia No It

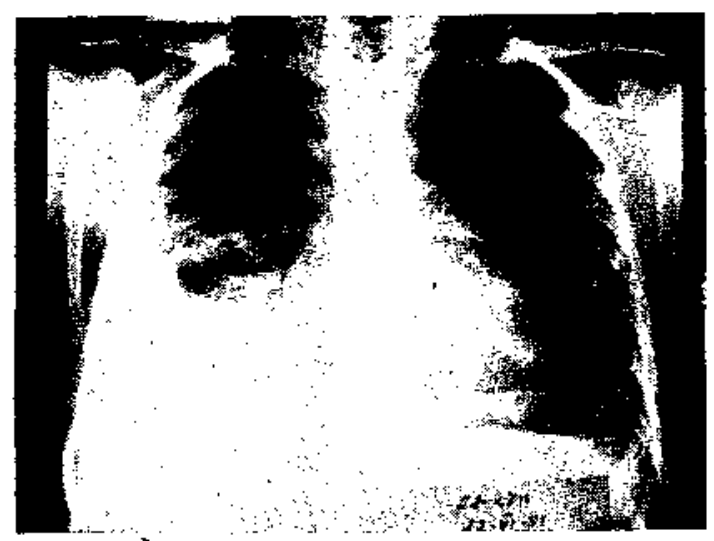

Radiografia No 12

Tcresa Corrca. Obs. 5L/34. Edad: 14 años. Ingresa al Hospital to 3 de enero del $5 \mathrm{~L}$, con $\mathrm{l}$ diagnóstico de TBC: pulmonar de rcinfección, modcradamente avanzada, caverna crónica del lóbulo supcrior izquierdo, activa. Koch demostrado (radiogralia No 14).

Su Icsión data de seis rucses alrís. habienclo recibido ya 80 grs. de Estreptomicina indicada por médico particular. Se practica una nuesa cura con Estrepto-

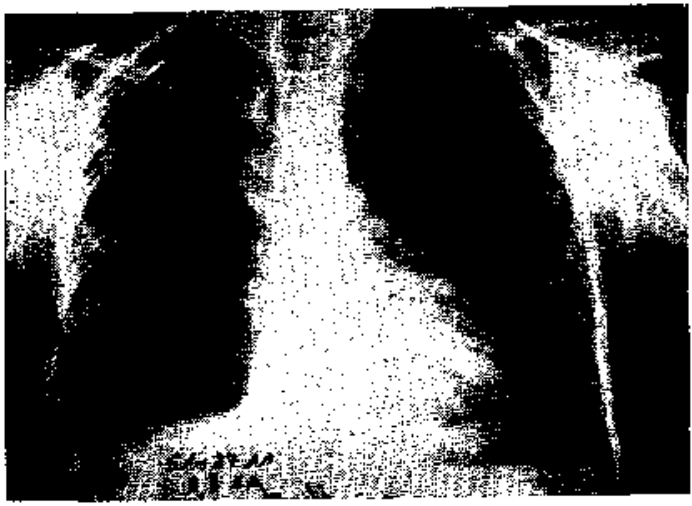

Ratiografia Ne
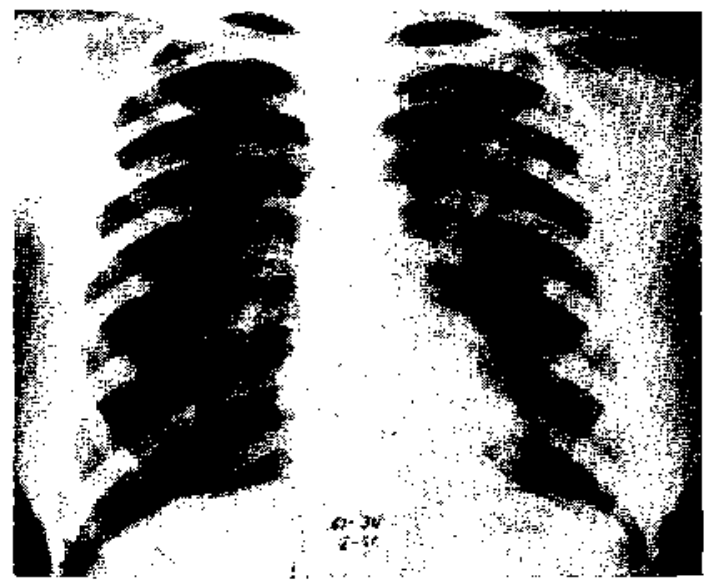

Radiografin $\mathrm{No}$ Ht

micina. P.A.s. y se inicia neumotórax. A pesar del buen colapso puImonar y broncoscopia negativa la caverna no mejora y continúa eliminando bacilo de koch. En este caso no sc indicó toracoplastía, porque si bicn la caverna era ricl lotuto stperior, se necesitaba sacar muchas costillas para poderla colapsar.

k. 19 de julio de 1951 se practica lobectonia superios izpuictala sin complicaciones.

El examen histopatológico revela una caverna con gricsas parcules fibrosas, cascosis en la superficie interna $y$ en el parćncuima vecino mábulos fibrocastosos.

Lltimo control un año trcs meses después: esti clínica y radiolóficamente saná (radiografía No 15).

I a causa de la muerte inmediata en el posoperatorio ba sido lit siguiente: en dos casos se produjo al 29 o 3.er día uná fístula bronquial con derrame pleural purulenta en uno y sanguinca en otro. En este caso se produjo a traves de esa fistula bronquial una inundación sanguínea del lado opuesto con asfixia y en el otro una diseminación broncógena tbc del lado sano. 


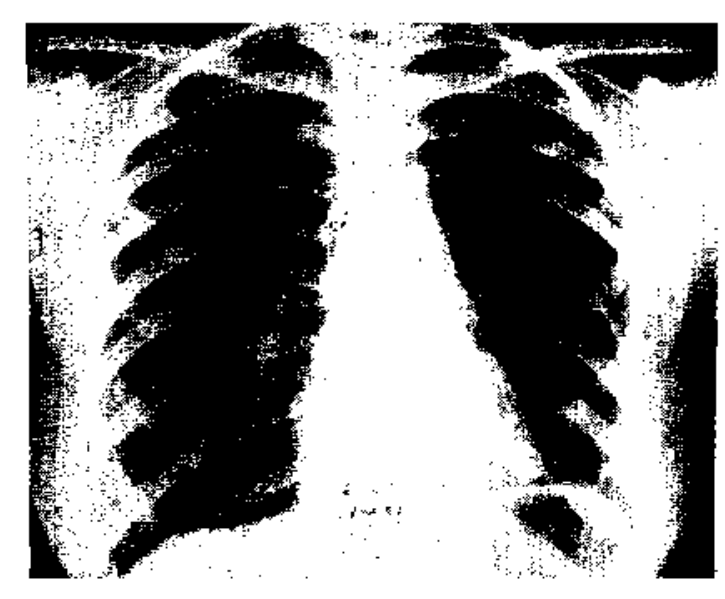

Kadiogratia 3015

Estos dos casos corresponden a neumonectomia por extensos procesos caseosos, neumonia caseosa que se extendía hasta la región hiliar donde existian ganglios que dificultaban consirlerablemente la intervención, ya que no se podía aislar los elementos del hilio. Fn otro caso la muerte se debic a una trombosis aurículo-ventricular derecha con infarto hemorrá. gico pulmonar. Tambićlu en este niño el raseo abarcaba toda la región hiliar y se practicó una lobertomía del lob. medio inferior.

El 43: caso fallecido en el postoperntorio fué un niño que tenía una neumonía caseosa y que sólo se alcanzó a hacer una toracotomía. Al ábrir el tórax se comprobí un bloque caseoso del loh. superior y netio. Al quererlo separar de lats adherencias se abrió una caverna en la pleura y al mismo tiempo se produjo una inmndación del Jado opuesto con atelectasia $y$ asfixia. Fallece al 5 e dia.

El 50 caso latal conesponde a una lobectomía inferior izala. por proceso filsroso caseoso ulcerado. En el acto operatorio se comprueban nodulos caseosos en el lob. sip. izclo. En el postoperatorio se produre una reartivación ipsolateral on el lob. sipr. izdo. y diseminación broncógena del laclo opuesto. Posteriormente se constituyc una fistula broncocutinea izquierda. Fallece a los 6 meses de operada.

lista paciente habja recibido ances de su ingreso $150 \mathrm{gr}$. de estreptomicina y P. $\mathbf{~} \mathrm{S}$, por lo que cremos que existia resistential a estas drogas. A esta causa aribuimos el cracaso de estos antibióticas en el postoperatorio.

El resto de los niños con resección, o sea 16 , están en perfectas condiciones hasta el momento. El tiempo de observación es en 3 de más de dos años, en 1 un añ $y$ ynedio, en 5 más de seis meses $y$ ell 4 menos.
Cuatro de estos niños presentaron compliraciones que fueron perfectamente controladas por los antibióticos usados conjuntamente, con los quimioterípicos. En estos niños la hidrazida clel ácido nicotínico no había sido usada anteriormente, hecho que fué decisivo en la mejoria.

Tres fueron complicaciones inmediatas postoperatorio: en un niño en forma de una diseminación broncógena al lado opuesto dando lugar a una infiltración mixta, infiltrativa atelectásica con bacilos de Koch.

En otro en forma de una reactivación de nóslulos que existían en el pulmón opuesto y en el 3.er caso con un derrame sanguíneo que se infectó con gérmenes banales dando lugar a cmpiema que cura al drenarse. La complicación tardia se produjo a los 6 meses en un niño neumonectomizado en forma de reactivación de nódulos que existian al lado opuesto.

En el resultado de estas intervenciones no sólo debemos considerar la curación delinitiva de sus focos tbc sino que habrá que estudiar si el parénquima pulmonar con que han quedado estos niños es suficiente para permitirles hacer una vida activa; en otras palabras nos parece de gran importancia estudiar qué alteraciones fisio-patológicas se producen en casos de neumonectomía y lobectomia y si éstas se producen qué importancia van a tener en el futuro del niño.

Se sabe por la experiencia de adultos que cuando se extrae una parte del pulmón, el resto del parénquima trata de ocupar el espacio dejado por éste, producićndose algunas veces, procesos de enfisema que naturalmentc deben comprometer, según su intensidad, en cierto grado la funcion de dicho parénquima.

En caso de lobectomía nos parece que esto no dlebe tener mucha importancia y es lo que nuestra reducida experiencia nos ha demostrado. El problema es distinto y se presenta más scrio cn casos de neimonectomía. En estos casos se observa que el pulmón sano trata de ocupar a travis de Inernias del mediastino, el sitio del pulmón resecado. Se comprende que este desplazamiento puede originar. Iuera de altetaciones vasculares o de otra índole del mediastino, intensas alteraciones enfisematosas del único julmón; por lo cual se puede comprometcr seriamente su función vital.

Para estos desplazanientos, los tisiólogos de adulto aconsejan en caso de neumonectomía rellenar el espacio dejado por toracoplastías amplias. Otros aconsejan vellenarlo con sangre o elementos menos peligrosos. 
De nuestros 5 casos de neumonectomía que están vivos sólo uno tiene un período de ob. servación de 1 año 3 meses. Las otros \& sólo 2,8 y 7 meses. En el primero se ha producido una hernia del mediastino con desplazamiento del pulmón sano al lado opuesto. No presenta ningún signo funcional de insuficiencia respiratoria, pero no sabemos qué importancia va a tener en el futuro. De los otros 3 presentaban antes de operarse un fibrotórax con sus mediastinos fijos por las adherencias y hasta el momento no se aprecian desviaciones. El 50 caso se hizo junto con la neumonectomía izda. una freno-exeresis que ha provocado un ascenso del henidiafragma izquierdo hasta cerca de la espina del homóplato impidienco el desplazamiento del pulmón opuesto. La importancia del desplazamiento lo dará la evolución futura, ya que nuestra observación tiene muy poco tiempo de control.

Ante el peligro de este desplazamiento nos parece que está indicado en nuestros futuros casos de neumonectomia colocar en el espacio que deja el pulmón, ya que en niños pequeños no debe hacerse toracoplastía, algún material plástico para lo cual hemos pensado en las bolitas de lucita.

\section{Gonclusiones}

1a Se estudial someramente el estado epidemiológico de la tbc. por que atraviesa nuestra población, recalcando sus fases de tuberculinización máxima, con las curvas de infección y morbomortalidad, demostrando que nuestra infancia se pone en contacto con el bacilo de Koch desde temprana edad, siendo la tbc. una de las principales causas de mortaliclad inlantil.

20 Se demuestra que el pronóstico de la tbc. infantil grave, abierta, es a través de 10 años de observacion, a pesar de todos los adelantos molernos muy desfavorable, oscilando su mortalidad entre 60 a $95 \%$.

30 Se estudian, pará mejorar este pronóstico, las posibilidades de la cirugía toráxica en la tbc. grave infantil. Se relata la experiencia de 47 casos de operaciones, siendo 31 por thc. activía y 16 en casos inactivos.

En los casos de tbc. activa se ha usado en 8 enfermos toracoplastín, en 2 neumolisis extrapleural con plombaje de lucita y en 21 casos resecciones.

40 Se insiste que la cinıgía está indicada solamente en los procesos tbc. irreversibles (caverna, procesos fibrocaseosos, campos de indu- ración, dilataciones bronquiales, etc.) en las cuales la colapsoterapia y antibióticos han fracasado.

50 Se estudian las indicaciones de las distinLas intervenciones en la edad adulta $y$ se precisa a través de nuestra experiencia cuáles son istas para la edad infantil.

60 El resultado de nuestra experienciá demuestra que la cirugía toríxica hecha por cirujanos y anestesistas expertos constituye un gran recurso para mejorar estas formas irreparables de tbc. activa o inactiva.

79 Siendo nuestro período de observación de los operados todavía reducido, no se pueden sacar conclusiones definitivas, sólo porlemos hablar de resultados inmediatos y de una observación que oscila entre "2 meses a 4 años.

En nuestro material de 47 casos hemos tenido una mortnlidad de 6 casos, siendo el estado actual de los vivos muy satisfactorio. El pronóstico definitivo de ellos, relacionado tanto a su proceso tbc., romo it la capacidad funcional de su aparato respinatorio, se verí sólo a tratvés de mís años de observación.

Como romplicaciones las más graves las constituyen las fistulas postoperatorias. Las diseminaciones hroncógenas y reactivaciones que son las otras complicaciones pueden ser controladas por los antibióticos y quimioterápicos. siempre que estos enfermos se operen antes que pierdan lit sensibilidad a esta droga.

Las fístulas bronquiales se producen especialmente en casos en que el proceso caseoso grave llega hasta la región hijiar y la engloba.

\section{NHIIOGRARIA}

BOHHXIVC, F: KIAKI, K-Die Offene Lungentubextintase bui Kincleme und Jugendlichen.

KI.1RF., K.-Die Prognose der oflener J.ungentuberkuIose trei Kinder und Jugendtichen.

VItL, B.-Fpideniologia de la tuberculosis en Chilc.

PFREDA, F.-Algunos aspectos de da Epixlemiología de la tuberculosis on la Comuna de Quinta Nommal. Boletin Méclico Sncial. Ano XVI, No 176 .

ARAYA. Y.-Epidemiologia te la Tuberculosis del Niio y algunos aspectos inmuno-biológicos y clinicos de la vacumacicin con BC. B. Bolctín Mídico Social. Año XVIT, No 156.

BRIJNER, it,-ludikationerim zt. Iobcktomic und Pneumonckomic Schwcizcriche Zcitshrift fur. tuberkulose fasc. 1, 19t\%).

OVERHOLT, R.; WILSON, N. Y GEHRING, Li-The place of pulnonary resection in the treatment of Tuberculosis. Jis. of. Chest 21:32.51J (jan.). 52.

ItII.SON, N. OVERHOLT, R.-Mrjot Surgical Iroseltures in the treatinent of Pulumonary Tuberculosis; in the Fundamentals of Pulmonary Tuberculosis. Thomas. 3949. 